\title{
SETTING STANDARDS AND INNOVATIONS
}

Dr. V. N. Mahalakshmi,

Vice Principal (Curriculum),I

Sri Balaii Vidyapeeth - Mahatma Gandhi Medical College and Research Institute Campus Pillaiyarkuppam, Puducherry - 607403, INDIA

\section{INTRODUCTION}

As a part of the quality initiatives involving TeachingLearning-Evaluation systems, the Exam division of Sri Balaji Vidyapeeth University, under the able guidance of our honorable Vice-Chancellor, Prof. K.R. Sethuraman, an educationalist par excellence, has taken initiatives to benchmark standards and innovations in the evaluation process.

\section{INNOVATIONS IN THE EXAM PROCESS}

The process of standardization started with an analysis of the strengths and weaknesses of the existing system and search for technically and logistically viable options. Once the analysis of the strengths and weaknesses of the existing system was complete, the following innovations/standardizations are introduced in the exam process.

\section{UG EXAMS}

1. Eliminating Ambiguity in questions - As a part of the quality initiatives, the SBVU exam division is set about eliminating ambiguity in questions. The question papers pattern is changed to include very short objective questions, focused short answers and structured essay questions. SBVUAHEAD, the Academy Of Health Education And Development, organized and conducted workshops on "Question paper setting and validation" to all faculty of the constituent colleges. As a follow-up of this work shop, all the Departments prepared the blue print guidelines of question papers based on the specific learning objectives (SLO) in mind. This is used as a template for the question paper setters.
- The paper setters are given elaborate blue print guidelines specific to the learning objectives of the subject being evaluated and also weightage for various systems. They are asked to fill in an item card analyzing the distribution of questions and marks across systems, level of difficulty and type of knowledge being tested recall, understanding or application. They are also asked to submit a relevant answer key covering the key points to be discussed in the answer. The paper setters are asked to frame specific and relevant questions, without ambiguity.

2. Vetting of the question papers -The question paper received from the examiners are subjected to a 3 stages vetting process involving,

- Matching against the blue print guidelines \& Looking for grammatical errors, errors in the framing of questions and repetition - to be done by COE's office.And also

- Checking for relevance and validity by the concerned subject expert.

- All question papers are subject to scrutiny and approval by the Vice- Chancellor, for their global relevance and applicability to the student population being tested.

A checklist encompassing these details is evolved and is being filled by the exam division.

3. The actual conduct of exams

- Secure online transmission and printing of question papers to off campus institutions in 
secure, encrypted form $1 / 2$ an hour before the start of exams.

4. Redesign of Answer Booklets - To expedite the exam process, OMR coding of answer books is introduced to code student and exam information. Each booklet has an unique barcode to tag examinee information and valuation.

5. The answer booklets are subjected to central evaluation. To eliminate 'examiner bias', each examiner is asked to correct the same question in all the answer booklets - Item Marking.

6. Post validation of learning outcomes is done through Evaluation of the group performance by plotting of the marks scored by all the students in the group to total marks awarded for each question. This process can identify the potential faults in the evaluation tool i.e., the question themselves or the evaluation process (examiners). This also helps in the need for moderation of the group score if there are flaws in the system / tools. Also, the triple feedback loop helps the curriculum planners to evaluate the validity of the learning process.

- Though this system has been used extensively in many Universities \& Colleges across India, our evaluation is unique, that it allows analysis of marks awarded to individual questions. This robust system was conceived and developed by Dr.Mahalakshmi under the able guidance of our Vice- Chancellor, Prof.K.R. Sethuraman, in collaboration with the Department of Information Technology to suit our local needs and has an error free rate of six sigma.

\section{PRACTICAL / CLINICAL EXAMS}

Having restructured the theory exams and systemized the exam process, we set out to objectivise the practical / clinical / oral exams. This is by introducing OSCE / OSPE and structured viva in the exams. A series of workshops on Objectivising the clinical /practical /Oral exams on OSCE / OSPE for faculty of the constituent colleges planned for in Feb-June 2014 and robust OSCE / OSPE blueprints and subject wise question banks developed. A national level, nodal meeting was held to present the SBV's plan to align with MCI and seek experts opinion on the project, in May 2014. The model blueprints with the expert groups' comments have been submitted to MCI for approval.

\section{ORAL EXAMS}

The oral / viva-voce exams for UG students have been restructured as 'Structured Viva' examinations, which follow a definitive blue print and specific mark allocation pattern for different topics.

The successful introduction of changes in the MBBS exams has helped us to carry forward the changes in the UG Dental \& Nursing courses.

\section{PG EXAM REFORMS}

The following innovations/changes were brought about in the PG exam process.

i. To eliminate ambiguity in the setting up of question papers, detailed blue printing of the question papers has been done and is followed.

ii. The long essay type questions are replaced by '10 Brief Essay' questions, to improve the sampling.

iii. To eliminate bias, all answer sheets of all students are being evaluated by all the examiners ( 4 times valuated)

iv. The evaluations of PG dissertations have been made more objective by using a designing checklist for examiners to evaluate the dissertations. The data from the evaluators is shared with the subsequent batch of PG students in the 'Dissertation Writing' workshops, so that such shortfalls can be minimized and eliminated. 